\title{
Extremely well-differentiated adenocarcinoma of the stomach: diagnostic pitfalls in endoscopic biopsy
}

\author{
Jongwon Lee ${ }^{1}$ In-Seob Lee ${ }^{2}$, Ji Yong Ahn ${ }^{3}$, Young Soo Park ${ }^{1}$, Jihun Kim ${ }^{1}$ \\ Departments of ${ }^{1}$ Pathology, ${ }^{2}$ Surgery, and ${ }^{3}$ Gastroenterology, Asan Medical Center, University of Ulsan College of Medicine, Seoul, Korea
}

\begin{abstract}
Background: Extremely well-differentiated adenocarcinoma (EWDA) is a deceptively bland-looking adenocarcinoma of the stomach. It often causes diagnostic problems, especially in endoscopic biopsy samples. To better recognize this deceptively bland lesion, we carefully reviewed a series of EWDAs treated at our institution. Methods: A total of 55 specimens from 19 patients were obtained. Endoscopic, gross and microscopic features defining EWDA were described and documented. For comparison, hyperplastic polyp specimens were randomly selected and analyzed. Results: Most cases (18 of 19, 94.7\%) were advanced gastric cancer (AGC) and primarily located in the body of the stomach (15 of $19,79.0 \%)$. The majority of AGCs were non-ulcerated $(11$ of $18,61.1 \%)$ with an undermining growth pattern and a relatively small mucosal involvement. Specific histologic features included an irregular glandular shape, an undulating apical cytoplasmic border, disproportionately large glands, a variably distended mucinous cytoplasm. Classical features, such as small infiltrating glands or desmoplastic reactions, were barely observed. Identification of irregularly spaced nuclei and disruption of the foveolar epithelial structure, along with atypical features described above were helpful in making a diagnosis especially in gastric forceps biopsies. Conclusions: Awareness of the histomorphologic characteristics described in this report would lead to timely diagnosis and prevent repeated endoscopic procedures.
\end{abstract}

Key Words: Stomach neoplasms; Adenocarcinoma; Missed diagnosis

Received: August 26, 2021 Revised: October 8, 2021 Accepted: October 12, 2021

Corresponding Author: Jihun Kim, MD, Department of Pathology, Asan Medical Center, University of Ulsan College of Medicine, 88 Olympic-ro 43-gil, Songpa-gu, Seoul 05505, Korea

Tel: +82-2-3010-4556, Fax: +82-2-472-7898, E-mail: jihunkim@amc.seoul.kr

Extremely well-differentiated adenocarcinoma (EWDA) of the stomach is a rare and an understudied neoplasm the diagnosis of which is especially challenging due to bland nuclear features and subtle architectural atypia. Intestinal- and gastric-type EWDAs are its subgroups, mimicking intestinal metaplasia and normal foveolar epithelium, respectively. Intestinal-type EWDAs consist of intestinal-type glands with various amounts of goblet and Paneth cells [1]. Gastric-type EWDAs are described as mucin-rich columnar cells with basally located, bland-looking nuclei mimicking hyperplastic foveolar epithelium or dilated pyloric glands [2].

Due to its deceptively bland morphology, misinterpretations of EWDA in gastric forceps biopsy are fairly common [2]. However, not many reports discuss its diagnostic histomorphology comprehensively. Therefore, we tried to extract applicable gross, histomorphologic features recurring in 19 EWDAs from our in- stitution assuming that EWDAs exhibit several indicative histological and growth patterns applicable in diagnostic approaches.

\section{MATERIALS AND METHODS}

\section{Case selection}

EWDA was defined as neoplastic glands comprised of highly differentiated cells mimicking intestinal metaplasia or normal gastric foveolar epithelium with mild nuclear atypia, according to the definition by Yao et al. [1], applied with slight modification. EWDA cases were identified and collected through routine clinical practice. In the screening process, the intestinal-type EWDAs presented as early gastric cancers (EGCs) with the wellknown crawling-type or shaking-hand-type morphologies were excluded. We focused more on the identification of advanced EWDAs that were relatively diagnostically urgent. The malig- 
nant nature was confirmed either by surgically resected specimens or by unequivocal clinical presentations as metastatic disease. The confirming procedure was warranted because the histologic appearances of EWDAs were not easily recognizable due to deceptively bland morphologies. After the confirmation of malignant nature, we collected all related pre-operative biopsy samples. Fourteen cases from the authors' institutional archive from 2018 to 2021 were collected by two pathologists (JL and JK).

To estimate the prevalence of EWDA, we reviewed 608 consecutive surgically resected advanced gastric cancers (AGCs) treated at our institution in 2010. AGCs were selected because most of the archived cases were AGCs. Five of them ( 5 of 608 , $0.08 \%$ ) fulfilled the criteria for EWDA, thus total of 19 cases was available for our study design. Patient characteristics, surgical and endoscopic findings with follow-up data were obtained from the medical records of Asan Medical Hospital.

\section{Endoscopic assessment}

Endoscopic data from available cases was collected and reviewed by expert gastroenterology specialist (JYA). The tumors were then classified as subepithelial-tumor-like lesions, slightly elevated or depressed lesions, or AGC Borrmann types according to the widely accepted endoscopic definition.

\section{Histologic assessment and statistical analysis}

Hematoxylin and eosin-stained sections for pretreatment biopsies and surgical specimens were available for all, and two pathologists (JL and JK) independently evaluated their gross findings, histomorphology, pathologic TN stage, and lymphovascular invasion statuses. Any discrepancy was resolved in consensus sessions under a multiheaded microscope. The ulcer proportion, defined as area of gross ulceration divided by the area of the entire tumor, were calculated in surgically resected ulcerated EWDAs and control group AGCs from the year 2010. We used a nonparametric test (Mann-Whitney) to determine the p-value for differences between the groups. Statistical analysis was performed using SPSS software ver. 18.0 (SPSS Inc., Chicago, IL, USA), with $\mathrm{p} \leq .05$ considered statistically significant.

All cases were evaluated and documented for the following histologic features observed in EWDAs (Fig.1, Supplementary Fig. S1): inharmonious disproportionate glands, irregularly shaped glands, undulating apical mucin border, and markedly distended mucinous cytoplasm. Inharmonious disproportionate glands referred to glands disproportionately larger than surrounding non-neoplastic glands. Irregular glandular shapes indicated irregular glandular structures that were cut off or distorted. Un- dulating mucin border described irregular, wobbly border of apical mucin caps. Markedly distended mucinous cytoplasm of EWDA cells indicated very large tumor cells sometimes exceeding 40 times the size of mature lymphocytes.

Background mucosa was also microscopically studied in terms of the presence of atrophic gastritis or intestinal metaplasia in adjacent mucosa. The time intervals between the initial biopsy and treatment, type of treatment, and the number of procedures performed were recorded. The original diagnoses of pretreatment biopsies were collected and compared with the final diagnoses.

\section{Immunohistochemical analysis}

Formalin-fixed paraffin-embedded tissue blocks were available for all. To determine the tumor immunophenotype, immunohistochemical staining was performed using antibodies against MUC-5AC (1:100, mouse monoclonal, clone MRQ-19, catalog No.292M-96, Cell Marque, Rocklin, CA, USA), MUC-2 (1:50, mouse monoclonal, clone Ccp58, catalog No. NCL-MUC-2, Novocastra, Newcastle upon Tyne, UK), MUC-6 (1:200, mouse monoclonal, clone CHL5, catalog No. NCL-MUC-6, Novocastra), CDX-2 (1:500, mouse monoclonal, clone EPR2764Y, catalog No. 235R-16, Cell Marque), c-erbB2 (1:8, mouse monoclonal, clone 4B5, catalog No. 790-4493, Ventana, Tusan, AZ, USA), p53 (1:1,000, clone DO-7, Dako, Glostrup, Denmark), Ki-67 (1:200, mouse monoclonal, clone MIB1, catalog No. M7240, Dako) and PTEN (1:100, rabbit monoclonal, clone 138G6, catalog No. 9559, Cell Signaling, Danvers, MA, USA). Expression of mucin core proteins and CDX-2 were investigated to help classification of tumors into gastric- or intestinal- subtypes. C-erbB2 immunohistochemistry was done to find out the potential therapeutic targets. We did p53 and Ki-67 immunohistochemistry to determine their helpfulness in identifying the EWDAs. Finally, PTEN immunohistochemistry was performed to confirm the PTEN protein expression loss in the case (case No. 3) with a certain PTEN mutation. All staining procedures were performed using a Ventana autostainer according to the manufacturer's instructions.

Cytoplasmic staining for mucin core proteins (MUC5AC, MUC2, and MUC6) and nuclear staining for CDX-2 were considered positive. C-erbB2 staining was evaluated based on traditional human epidermal growth factor receptor 2 (HER2) immunohistochemistry scoring guidelines [3]. Cases scored equivocal for $\mathrm{C}$-erbB2 were tested for HER2 gene copy-number by silverenhanced in situ hybridization. For scoring, we followed general guidelines for HER2 copy-number evaluation as described by Jeong et al. [4]. Two pathologists (JL and JK) independently scored 

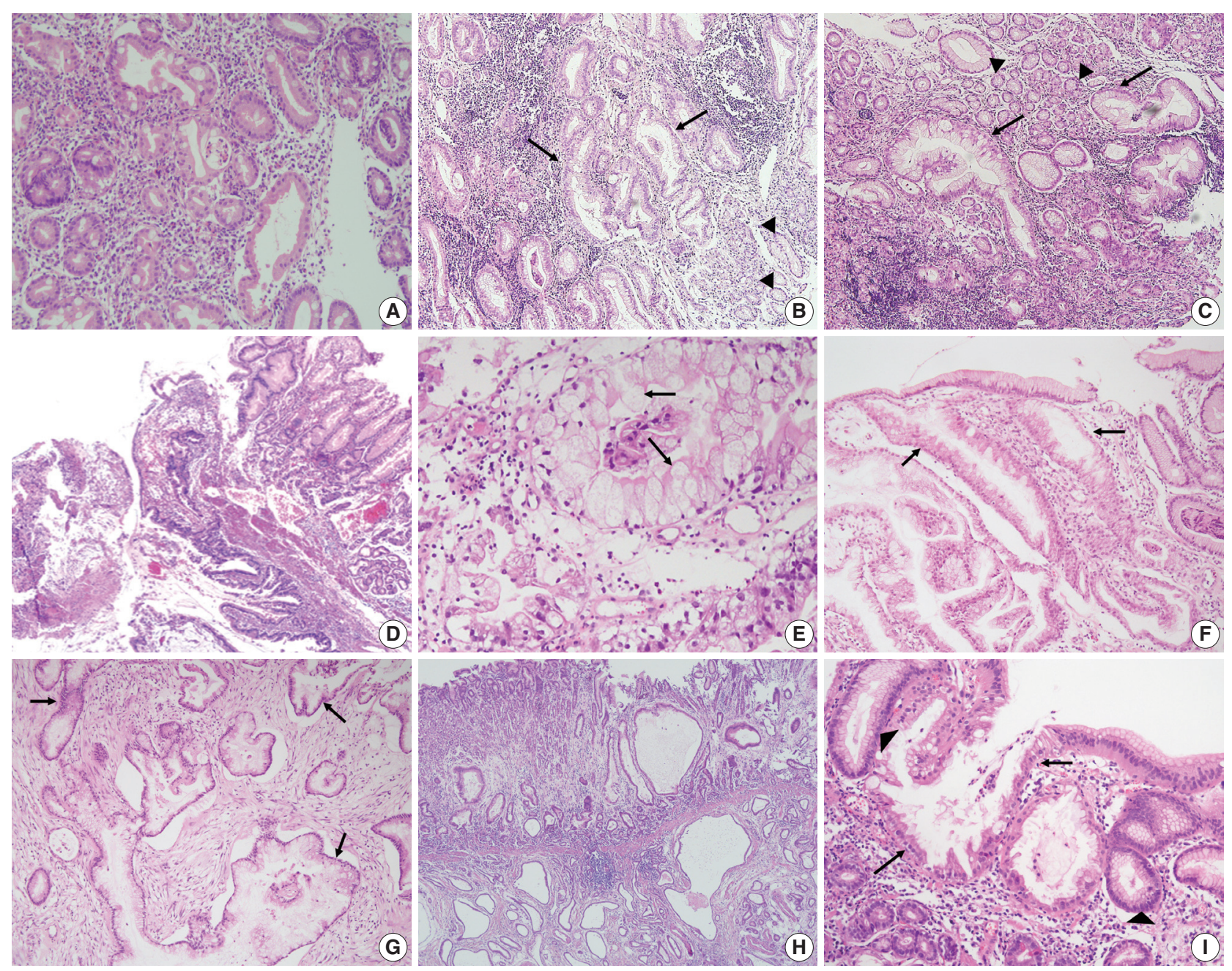

Fig. 1. Representative photomicrographs of extremely well-differentiated adenocarcinoma (EWDA). (A) Intestinal-type EWDA featuring goblet cells is observed in case No. 12. (B) Irregularly shaped glands (arrows) opposed to normal foveolar glands (arrowheads) are observed in case No. 4. (C) Inharmoniously large glands (arrows) are noticeable against benign foveolar glands (arrowheads) in case No. 4. (D) A focal mucosal opening giving way to larger, deeply seated glands is noted, resembling lobular endocervical glandular hyperplasia of the uterine cervix (case No. 17). (E) Extremely large neoplastic glands, at least 20 times the size of normal lymphocytes, are noted in case No. 2. An undulating apical mucinous border is also observed (arrows). (F, G) Case No. 3 shows bland-looking gastric-type EWDA glands (arrows) both in gastric $(F)$ and omental biopsy specimens $(G)$. (H) Case No. 11 shows cystically dilated thin neoplastic glands invading the muscularis propria with gastritis cystica profunda-like portions in the submucosa. (I) Glandular cancerization (arrows) in the background of normal foveolar glands (arrowheads) is more commonly found in EWDA than in conventional gastric carcinomas.

the immunostaining, and any discrepancy was resolved by consensus. Selective next-generation sequencing (NGS) data were available for three cases (case Nos. 2, 3, and 9). NGS was performed according to our routine clinical targeted cancer panel as described previously [5].

\section{RESULTS}

\section{Clinicopathologic features}

The clinical information of the 19 cases were listed in Table 1.
The median age of the patients was 63 years (range, 31 to 81 years) and the male to female ratio was 5.3:1 (16:3). The majority of the tumors (15 of $19,79.0 \%$ ) were located in the body of stomach in contrast to the usual type gastric carcinomas in which antral location is more common. The median tumor size in the resected cases was $4.0 \mathrm{~cm}$ in the greatest dimension (range, 2.2 to $10.0 \mathrm{~cm}$ ). Most of the cases were clinically or pathologically proven AGCs (18 of 19, 94.7\%).

Most patients were treated with surgery and adjuvant chemotherapy (11 of $19,57.9 \%)$. Surgery alone was performed in two 
Table 1. Clinical information of 19 cases of EWDA

\begin{tabular}{|c|c|c|c|c|c|}
\hline Case No. & Age (yr) & Sex & Treatment & Follow-up (mo) & Status \\
\hline 1 & 77 & $\mathrm{~F}$ & ESD & 17 & NED \\
\hline 2 & 66 & $F$ & Chemotherapy & 19 & AWD \\
\hline 3 & 31 & M & Chemotherapy & 0.5 & DOD \\
\hline 4 & 56 & $\mathrm{~F}$ & Surgery ${ }^{a}$ & 35 & AWD \\
\hline 5 & 60 & M & Surgery ${ }^{a}$ & 28 & NED \\
\hline 6 & 62 & M & Surgery ${ }^{a}$ & 23 & NED \\
\hline 7 & 39 & M & Surgery ${ }^{a}$ & 26 & NED \\
\hline 8 & 67 & M & Chemotherapy & 14 & DOD \\
\hline 9 & 72 & M & Surgerya & 26 & AWD \\
\hline 10 & 69 & M & Surgery ${ }^{a}$ & 16 & NED \\
\hline 11 & 58 & M & Surgerya & 8 & NED \\
\hline 12 & 61 & M & Surgerya & 15 & NED \\
\hline 13 & 48 & M & Chemotherapy ${ }^{b}$ & 13 & NA \\
\hline 14 & 63 & M & Surgery ${ }^{a}$ & 80 & NA \\
\hline 15 & 79 & M & Surgeryc & 121 & NA \\
\hline 16 & 65 & M & Surgery ${ }^{a}$ & 70 & NA \\
\hline 17 & 81 & M & Surgerya & 79 & DOD \\
\hline 18 & 55 & M & Surgery ${ }^{c}$ & 15 & NA \\
\hline 19 & 66 & M & Surgery ${ }^{c}$ & 15 & NED \\
\hline
\end{tabular}

EWDA, extremely well-differentiated adenocarcinoma; F, female; ESD, endoscopic submucosal dissection; NED, no evidence of disease; AWD, alive with disease; M, male; mo, months after diagnosis; DOD, died of disease; NA, not available (cannot be assessed).

aSurgery and adjuvant chemotherapy; ${ }^{b}$ Chemotherapy and metastatectomy (right hemicolectomy); 'Surgery alone. patients ( 2 of 19, 10.5\%). Four of the five initially metastatic cases received chemotherapy without gastric resection ( 4 of 19 , $21.1 \%)$. Endoscopic submucosal dissection was performed on the EGC (case 1, 1 of 19, 5.3\%). The majority of the surgically resected AGC cases infiltrated to the subserosa (pT3, 7 of 14, $50.0 \%$ ). The other cases penetrated to the serosa (pT4a, 4 of 14, $28.6 \%$ ) or invaded muscularis propria (pT2, 3 of $14,21.4 \%$ ). The EGC case invaded the submucosa (pT1b). Lymphovascular invasion was present in some resected specimens including the EGC case (5 of 15, 33.3\%). Nodal metastasis was histologically identified in some of the surgically resected cases ( 5 of $15,33.3 \%$ ). Only one (case No. 14) of such cases showed a very minor $(<1 \%)$ component of moderately differentiated adenocarcinoma. Even in the metastatic lymph nodes, the tumors retained their extremely well-differentiated morphology.

Patients were followed up for variable time intervals, ranging from 0.5 to 121 (median, 19 months) (Table 1). Some patients were alive without evidence of disease at last contact (8 of 19), $42.1 \%$ ), while others were lost to follow-up ( 5 of $19,26.3 \%)$. Of the five patients with distant metastasis at the time of diagnosis, the majority died of the disease at 5 months, 12 months, and 15 months after initial chemotherapy (3 of 19, 15.7\%). The others were alive with disease at last contact ( 3 of $19,15.7 \%$ ).

Table 2. Pathologic and endoscopic information of 19 cases of EWDA

\begin{tabular}{|c|c|c|c|c|c|c|c|c|}
\hline Case No. & $\begin{array}{l}\text { Tumor size, } \\
\text { greatest dimension } \\
(\mathrm{mm})\end{array}$ & Location & $\begin{array}{l}\text { Endoscopic } \\
\text { impression }\end{array}$ & $\begin{array}{l}\text { Mucosal } \\
\text { ulceration }\end{array}$ & $\begin{array}{l}\text { Macroscopic } \\
\text { finding }^{\mathrm{a}}\end{array}$ & $\mathrm{T} / \mathrm{N}$ & $\begin{array}{l}\text { Distant } \\
\text { metastasis }^{b}\end{array}$ & $\begin{array}{l}\text { Lymphovascular } \\
\text { invasion }\end{array}$ \\
\hline 1 & 33 & Body & SET & Absent & EGC lla & $\mathrm{T} 1 \mathrm{~b} / \mathrm{Nx}$ & Absent & Present \\
\hline 2 & NA & Body & SET & Absent & Borrmann 1 & $\mathrm{~T} 2 / \mathrm{N}^{\mathrm{b}}$ & Present & NA \\
\hline 3 & NA & Body & SET & Absent & Borrmann 1 & $\mathrm{~T} 3 / \mathrm{N}+$ & Present & NA \\
\hline 4 & 50 & Body & Slight elevation & Absent & Borrmann 3 & T3/N1 & Absent & Absent \\
\hline 5 & 25 & Body & Slight elevation & Absent & Borrmann 1 & T4a/NO & Absent & Present \\
\hline 6 & 40 & Cardia & Typical Borrmann 3 & Present & Borrmann 3 & T3/N3a & Absent & Absent \\
\hline 7 & 22 & Body & Slight elevation & Absent & AGC mimicking EGC type lla & T3/NO & Absent & Absent \\
\hline 8 & NA & Body & Borrmann 4 & Absent & Borrmann 4 & $\mathrm{~T} 3 / \mathrm{N}_{+}$ & Present & NA \\
\hline 9 & 100 & Body & Slight elevation & Absent & Borrmann 4 & T3/NO & Present & Absent \\
\hline 10 & 40 & Body & Slight elevation & Absent & Borrmann 3 & T3/NO & Absent & Present \\
\hline 11 & 33 & Body & Slight elevation & Absent & AGC mimicking EGC type lla & T4a/NO & Absent & Present \\
\hline 12 & 52 & Body & Slight depression & Absent & AGC mimicking EGC type Ilc & T4a/NO & Absent & Absent \\
\hline 13 & NA & Body & Borrmann 4 & Absent & Borrmann 4 & $\mathrm{~T} 2 \mathrm{~N}+$ & Present & NA \\
\hline 14 & 57 & Antrum & Typical Borrmann 2 & Present & Borrmann 2 & T3N2 & Absent & Absent \\
\hline 15 & 33 & Body & Typical Borrmann 3 & Present & Borrmann 3 & T2NO & Absent & Absent \\
\hline 16 & 65 & Body & NA & Present & Borrmann 3 & T4aN3 & Absent & Present \\
\hline 17 & 25 & Cardia & SET & Present & Borrmann 1 & T3N1 & Absent & Present \\
\hline 18 & 33 & Cardia & Typical Borrmann 3 & Present & Borrmann 3 & T2NO & Absent & Absent \\
\hline 19 & 30 & Body & Slight elevation & Absent & Borrmann 4 & T2No & Absent & Absent \\
\hline
\end{tabular}

EWDA, extremely well-differentiated adenocarcinoma; SET, subepithelial tumor; EGC, early gastric cancer; NA, cannot be assessed; AGC, advanced gastric cancer.

${ }^{a}$ Macroscopic finding was classified according to World Health Organization criteria; ${ }^{b} \mathrm{~N}+$, clinically assessed nodal metastasis. 

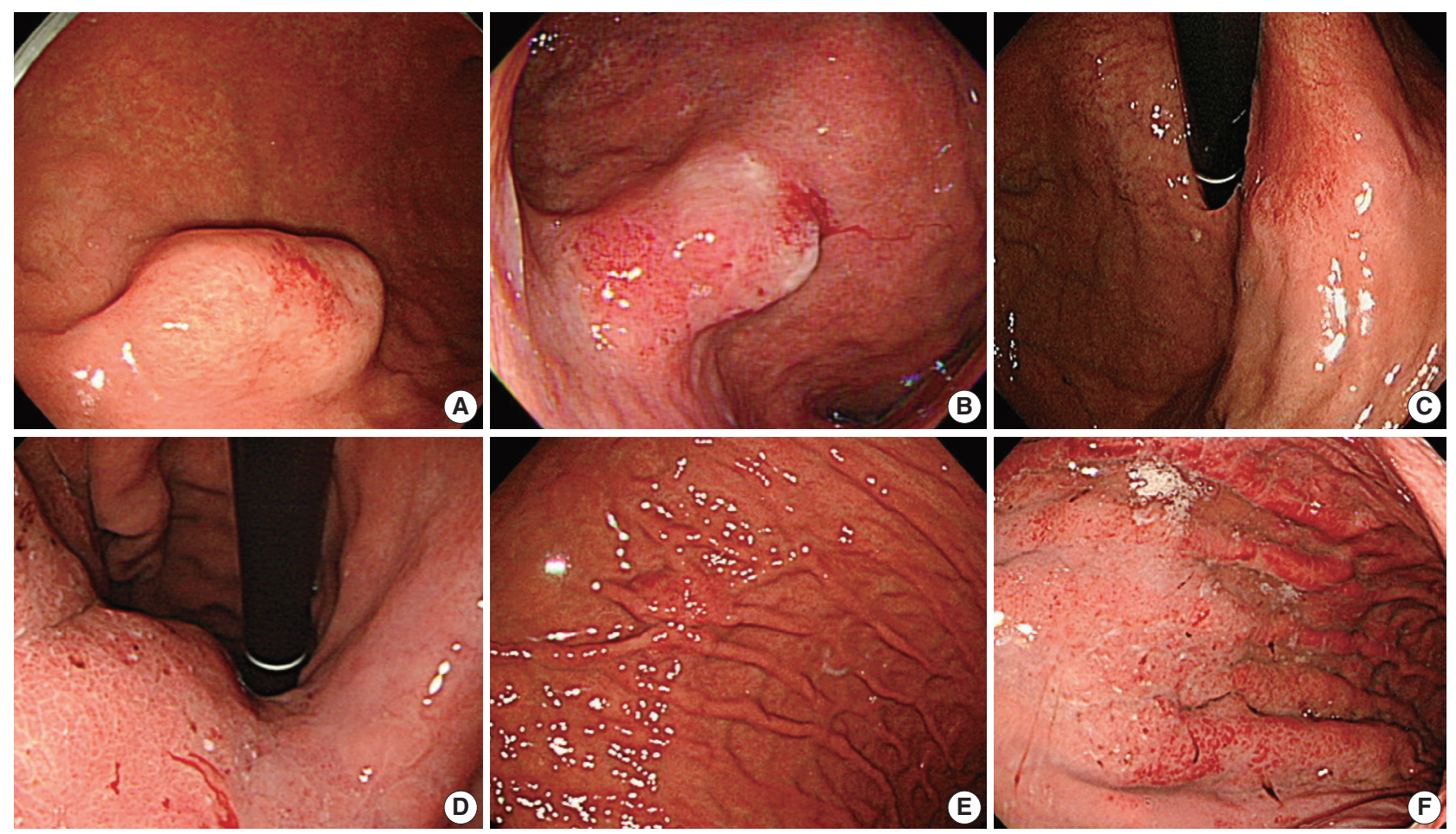

Fig. 2. Endoscopic appearances of diagnostically difficult extremely well-differentiated adenocarcinoma cases. (A) A mucosal elevation with mostly intact mucosa simulating a subepithelial tumor is noted (case No. 1). (B) A slightly elevated lesion with vascular engorgement is noted at angle (case No. 6). (C) A slightly depressed lesion is observed in case No. 12. (D) Diffusely thickened gastric wall in the gastric body is observed (case No. 9). (E) A slightly elevated mass is identified in case No. 7. (F) Thickened gastric folds with vascular engorgement (endoscopically Borrmann type 4) are seen in case No. 13.

\section{Macroscopic findings}

Eighteen cases with available endoscopic data were studied for endoscopic impressions (Table 2, Fig. 2). In two-thirds of the cases, malignant nature was not easily recognizable even by an experienced gastroenterologist (JYA) (Endoscopically assessed Borrmann type 3, 3 of 18 [16.6\%], Borrmann type 4, 2 of 18 [11.1\%], and Borrmann type 2, 1 of 18 [5.6\%]) (Fig. 2). Compared to usual gastric adenocarcinomas, EWDAs more frequently showed Borrmann type 4 and 1 lesions in surgically resected specimens (Bormann type 4, 4 of 18 [22.2\%] and Borrmann type 1, 4 of 18 [22.2\%]) with less frequent ulcer formation. Also, ulcer proportions of Bormann type 2 and 3 EWDAs were significantly lower than those of control group AGCs (mean value, $28.0 \%$ vs. $57.3 \%, p=.0048$ ) (Supplementary Table S1, Supplementary Fig. S2).

\section{Histologic assessment}

Most of the cases were histologically gastric-type (13 of 19 [68.4\%]), while the others were intestinal-type EWDAs (6 of 19 [31.6\%]) (Table 3). Irregular glandular shape was universally present (19 of 19 [100\%], pretreatment biopsy; 19 of 19 [100\%], surgically resected specimen), and inharmonious glands were present in the majority ( 9 of 19 [47.4\%], pretreatment biopsy; 11 of 19 [57.9\%], surgically resected specimen). About half of the cases showed undulating mucin borders (11 of 19 [57.9\%], pretreatment biopsy; 12 of 19 [63.1\%], surgically resected specimen) or distended mucins (8 of 19 [42.1\%], pretreatment biopsy; 9 of 19 [47.4\%], surgically resected specimen). Hyperplastic polyps showed distended mucin in some (6 of 19 [31.6\%]) but other descriptive findings of EWDA were not present. Intestinal metaplasias in the adjacent background mucosa tended to be more frequently observed in intestinal-type EWDAs than in gastric-type EWDAs (gastric-type EWDA, 4 of 13 [30.8\%] vs. intestinal-type EDWA, 4 of 6 [66.7\%], $\mathrm{p}=$.238) (Table 3).

Histologic characteristics worth mentioning in each case were separately recorded (Fig. 1, Supplementary Fig. S1). Case No. 2 exhibited exceptionally large cells with mucin distention, which were approximately 20 times the size of mature lymphocytes. Case No. 3 showed deceptively benign-looking cells with small nuclei, simulating normal foveolar gland epithelium. The same cells were also noted in the patient's omental biopsy indicating metastasis. In case No. 4, the neoplastic glands were cystically 
Table 3. Histologic and immunohistochemical features of 19 cases of EWDA

\begin{tabular}{|c|c|c|c|c|c|c|}
\hline Case No. & $\begin{array}{l}\text { Histologic } \\
\text { type }\end{array}$ & $\begin{array}{l}\text { Hyperplastic polyp } \\
\text { (biopsy) }\end{array}$ & $\begin{array}{c}\text { Histologic } \\
\text { features in } \\
\text { pretreatment biopsies }\end{array}$ & $\begin{array}{c}\text { Histologic } \\
\text { features in } \\
\text { surgical specimens }\end{array}$ & $\begin{array}{l}\text { Background mucosa } \\
\text { in pretreatment biopsies }\end{array}$ & $\begin{array}{l}\text { Background mucosa } \\
\text { in surgical specimens }\end{array}$ \\
\hline 1 & Gastric & $M$ & $S, U$ & $M, S, U$ & Atrophy & Atrophy \\
\hline 2 & Gastric & - & $M, S, U$ & NA & - & NA \\
\hline 3 & Gastric & - & $M, S, U$ & NA & - & NA \\
\hline 4 & Gastric & - & I, M, S, U & I, M, S, U & - & - \\
\hline 5 & Gastric & $M$ & $M, S, U$ & $\mathrm{M}, \mathrm{S}, \mathrm{U}$ & - & $\mathrm{IM}$ \\
\hline 6 & Gastric & - & $M, S, U$ & $M, S, U$ & - & - \\
\hline 7 & Intestinal & - & $S$ & $S$ & - & - \\
\hline 8 & Gastric & $M$ & I, M, S, U & NA & $\mathbb{I M}$ & NA \\
\hline 9 & Intestinal & - & I, S, U & I, S, U & - & - \\
\hline 10 & Gastric & - & I, S & I, S & - & $\mathrm{IM}$ \\
\hline 11 & Gastric & - & I, S & I, S, U & - & - \\
\hline 12 & Intestinal & M & $S, U$ & I, S, U & $\mathrm{IM}$ & $\mathrm{IM}$ \\
\hline 13 & Gastric & - & $S, U$ & I, M, S, U & $\mathrm{IM}$ & Atrophy, IM \\
\hline 14 & Gastric & - & I, M, S & I, M, S & - & $\mathrm{IM}$ \\
\hline 15 & Intestinal & - & $S$ & I, S & $\mathbb{I M}$ & $\mathrm{IM}$ \\
\hline 16 & Gastric & - & I, M, S, U & I, M, S, U & - & - \\
\hline 17 & Gastric & $M$ & $S$ & $S$ & - & - \\
\hline 18 & Intestinal & - & I, S & I, S & $\mathrm{IM}$ & $\mathrm{IM}$ \\
\hline 19 & Intestinal & $M$ & I, S & I, S & $\mathrm{IM}$ & $\mathrm{IM}$ \\
\hline
\end{tabular}

EWDA, extremely well-differentiated adenocarcinoma; I, inharmonious disproportionate glands; M, distended mucin; S, irregular glandular shape; U, undulating apical mucin border; NA, not available; IM, intestinal metaplasia.

dilated progressing towards the serosa. Case No. 11 showed mucosal cystic glands invading muscularis propria with gastritis cystica profunda-like morphology.

The epicenters of EWDAs were in the deeper mucosa or submucosa with infrequent ulcer formation. The overlying mucosa was without ulcer but involved by frequent glandular cancerization (Fig. 1I), or focal mucosal openings leading to large, deeply seated glands (Fig. 1D). The latter pattern was reminiscent of lobular endocervical glandular hyperplasia of the uterine cervix. It was also remarkable that desmoplastic reactions were barely observed.

\section{Differentiation from non-neoplastic foveolar glands}

Regarding differential diagnosis between gastric-type EWDAs and hyperplastic foveolar glands, we focused on three histologic features: (1) irregular glandular shape, (2) irregular spacing of nuclei, and (3) disruption of four lines. Irregular glandular shape was present in all EWDAs in contrast to hyperplastic polyps (Table 3). Irregular spacing of the nuclei was a distinct feature of EWDA, which referred to scattered nuclei not aligned to the basement membrane with disrupted polarity (Fig. 3). Four lines of the foveolar epithelium, formed by the apical mucin cap, base of the mucin cap, cytoplasm and nucleus [6] were disrupted or disappeared in EWDA glands.

\section{Molecular features}

All 19 cases were variably positive for gastric markers (Fig. 4, Supplementary Table S2). Positive MUC5AC and CDX-2 immunolabeling were closely associated with histological gastricand intestinal-type EWDAs, respectively. MUC6 and MUC2 expressions were not prominent in both subtypes, unlike the immunohistochemical profile of usual type gastric adenocarcinomas with diffuse expression of either markers. On an immunohistochemical basis, 13 (68.4\%) and six (31.6\%) cases showed gastric- and intestinal-phenotypes, identical to the histological classifications (Supplementary Table S2). Although p53 and Ki67 immunolabelings were mildly increased relative to normal foveolar epithelial cells, it was not distinct enough. Instead, their staining patterns were helpful because EWDAs showed diffusely increased staining patterns while benign glands showed locally increased staining in proliferative zones (Fig. 3E, F). C-erbB2 was equivocally expressed in two (case Nos. 2 and 13), but the silver in situ hybridization results were negative.

There also were a few cases with targeted cancer panel sequencing results (case Nos. 2, 3, and 9). A few notable mutations were found: NRAS G12D, STK11 Q220Pfs*38 (case No. 2), PTEN L108R and Y178C (case No. 3), and KRAS G12D (case No. 9) (Supplementary Table S3). Even though the functional significance of the PTEN L108R and Y178C mutations is not known, 
the loss of PTEN protein expression in this tumor (Fig. 4D) suggested at least one of the two PTEN mutations be loss of function mutation.
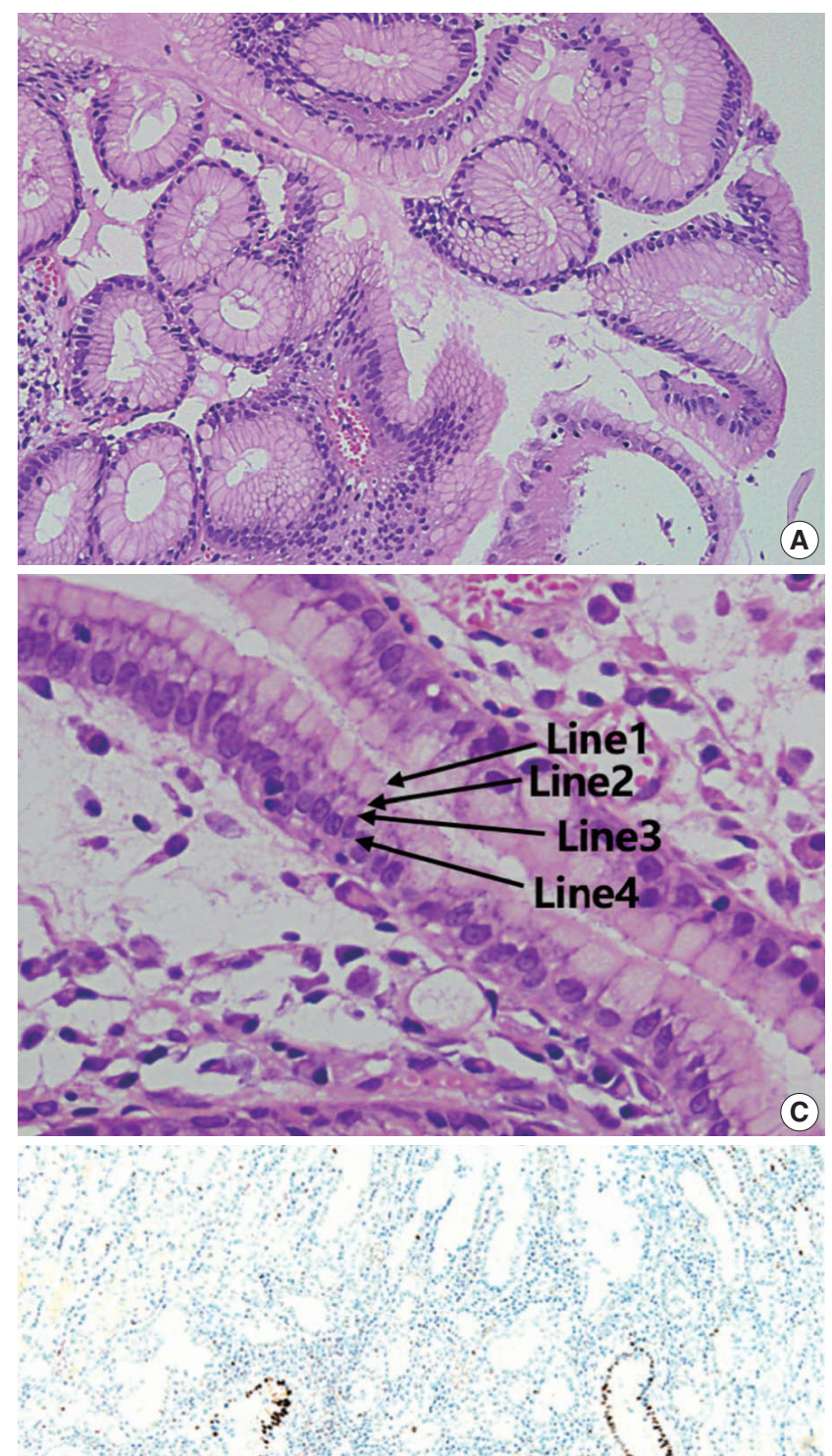

\section{Evaluation of pretreatment biopsies}

One to eight pre-therapeutic endoscopic examinations were performed in 19 patients (median, 2; mean, 2.5). A total of 55
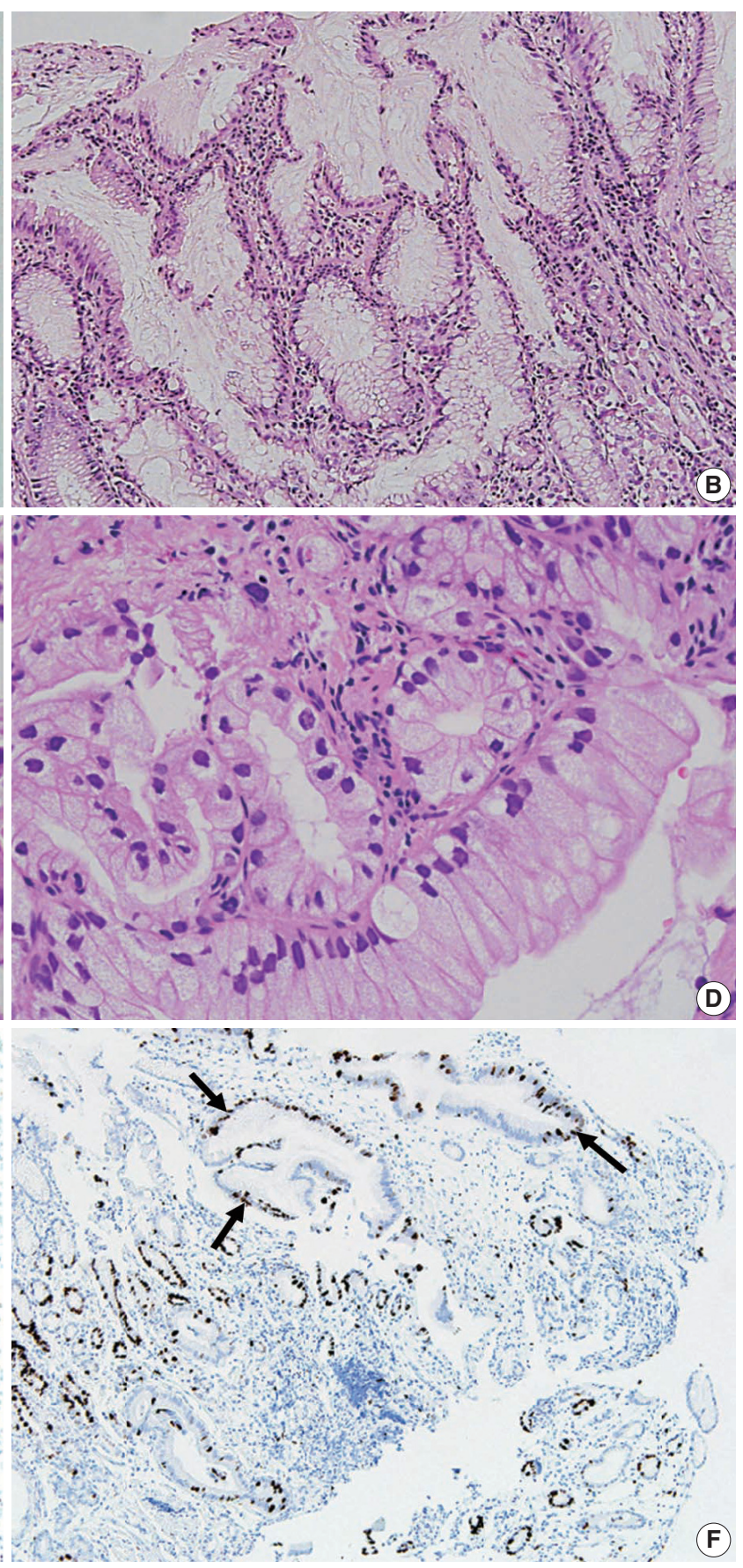

Fig. 3. Comparison between extremely well-differentiated adenocarcinoma (EWDA) and its mimickers. (A) Hyperplastic polyp shows an organized gland shape and aligned nuclei. (B) EWDA glands (case 4) show an irregular glandular shape and irregular nuclear spacing. (C) Normal foveolar epithelium displays regular nuclear spacing with maintenance of the "4 lines" (arrows): line 1, the gastric-type mucin vacuole; line 2, the base of the mucin vacuole; line 3, the cytoplasm; and line 4, the nuclei. (D) Case No. 6 shows large tumor cells with ample mucin, hyperchromatic nuclei, and disrupted four lines. (E) p53 expression is markedly increased in the tumor cells in contrast to the background foveolar epithelium of case No. 7 (p53 immunohistochemistry). (F) Ki-67 in normal foveolar epithelium shows increased expression only along the base of the crypts while the tumor glands (arrows) show a diffuse increase in case No. 8 (Ki-67 immunohistochemistry). 

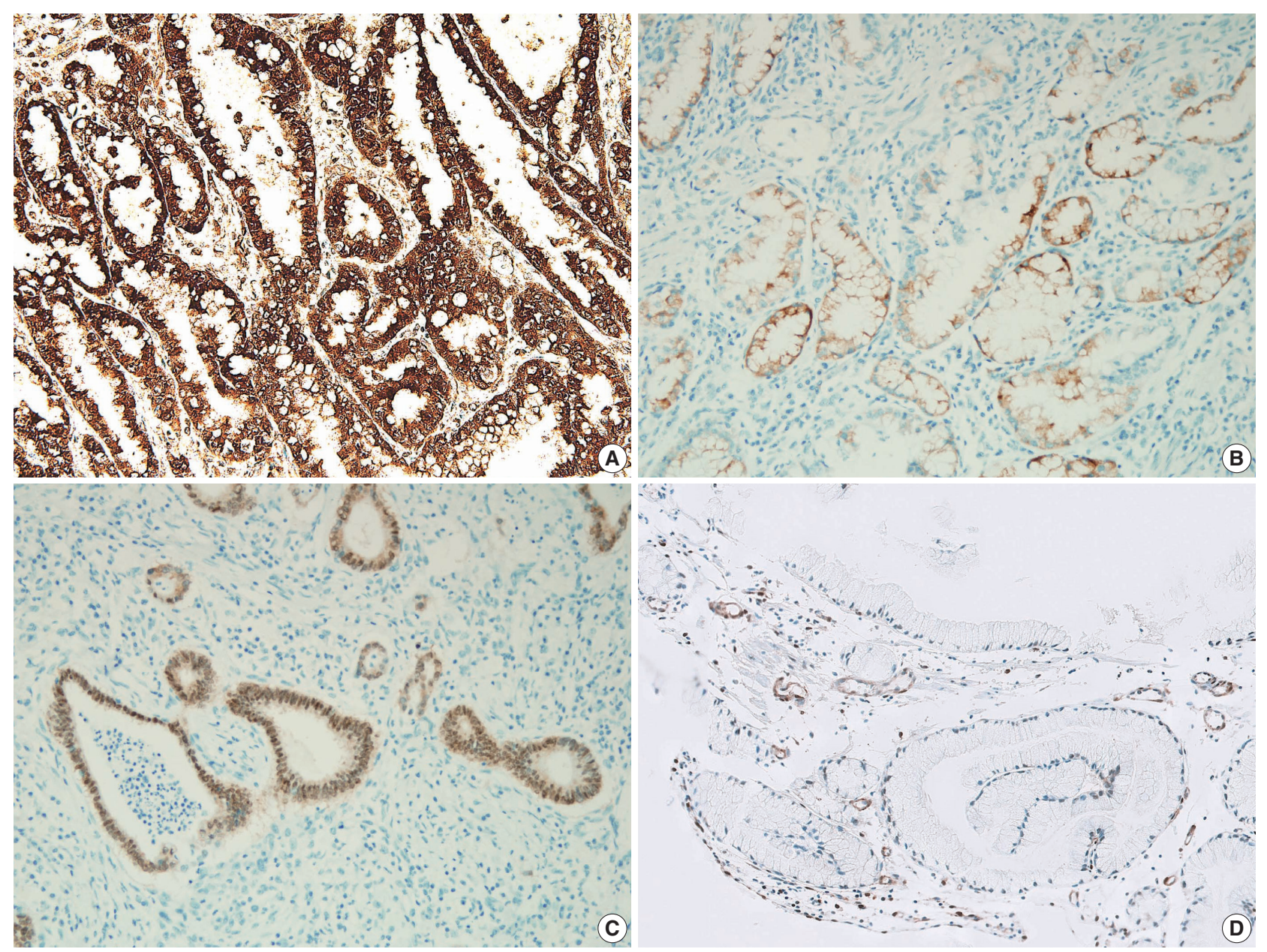

Fig. 4. Immunohistochemical pattern of extremely well-differentiated adenocarcinoma (EWDA). (A) Gastric-type EWDA expresses diffuse MUC5AC immunoreactivity in case 5. (B) MUC6 is focally expressed in case No. 5. (C) CDX-2 shows diffuse nuclear expression in an intestinal-type EWDA (case No. 7). (D) PTEN is lost in this PTEN mutant large cancer cells in contrast to the normal expression in endothelial cells in case No. 3.

tissue biopsies from 19 patients were available for review. The original diagnoses were 'adenocarcinoma' or 'suspicious for adenocarcinoma' in 46 biopsies ( 46 of 55, 84\%). Retrospective review of the remaining 11 specimens revealed that 10 of them were initially misdiagnosed (Supplementary Table 4).

\section{DISCUSSION}

EWDA consists of bland-looking malignant cells which is difficult to diagnose and less discussed in the literature. In our study, EWDAs were deep-seated tumors in the body of the stomach with less ulcer formation. It was also notable that endoscopically slightly elevated lesions or subepithelial tumor-like lesions were common, which is rarely suspected for malignancy by endoscopists $[7,8]$. Four histologic features including irregular glandular shape, undulating apical mucin border, and inharmonious glands and distended mucin were key histologic features of EWDA. In addition, irregular nuclear spacing and disruption of the four lines were helpful in discriminating of gastric-type EWDAs from hyperplastic foveolar glands. We believe that our study would help pathologists recognize this deceptively bland subtype of gastric adenocarcinoma.

Less ulcer formation of EWDAs could be explained by its undermining growth pattern. The findings were consistent with previous reports which mentioned EWDAs preferentially growing beneath the mucosa, forming polypoid masses [1]. Lobular endocervical glandular hyperplasia-like glands in the submucosa, also noted in other studies, were frequently observed accounting for frequent mucosal sparing of EWDAs [1,2]. Those findings suggest that some of the EWDA cases might have been originated from a deeper part of the mucosa.

Gastric-type EWDAs are especially cryptic because it simu- 
lates hyperplastic foveolar glands, and in many circumstances the distinction should be made in small gastric forceps biopsies. Undulating apical mucin border, along with irregular glandular shape, irregular nuclear spacing, and the disruption of four lines of foveolar epithelium, should strongly suggest EWDA especially in ambiguous endoscopic settings. Mucin distension, although present in many EWDAs, was sometimes seen in hyperplastic polyps and only marked mucinous distention sizing more than $\times 20$ that of adjacent lymphocytes would help in differential diagnoses. Inharmoniously large glands in EWDAs, as in our cases, also have been described in the literature [1,2], so atypically large or distinct glands should also raise concern for EWDA. Furthermore, even though intestinal metaplasias were rare adjacent to gastric-type EWDAs, its pathogenetic significance is yet to be discussed.

One helpful immunohistochemical marker might be Ki-67, because the labeling index was randomly increased in the carcinoma cells in contrast to reactive lesions with only basal cryptal increase, consistent with the findings by Niimi et al. [9]. Another useful marker might be $\mathrm{p} 53$ because its expression was increased against the background gastric foveolar epithelium in EWDAs [9], although null- or diffuse-type mutation pattern p 53 immunolabeling [10] was not identified. Treatment-wise, C-erbB2 expression or actionable genetic alterations were not found in any. Further studies for the therapeutic targets are recommended in the future.

One of the limitations to our study is that the control group of AGC cases were retrieved from a different time period, due to the extreme rarity of EWDA cases. Furthermore, systematic quantitative analyses could not be performed because our study was focused on the morphologic diagnosis of very rare EWDA cases. Also, selection bias towards AGCs occurred because unequivocally metastatic cases were selectively included in the study design. The referral bias as a tertiary medical institution also contributed. Furthermore, NGS results are available only in a small portion of our cases. Patients lost to follow-up also posed problems because prognostic data could not be gathered sufficiently. We suggest that proper epidemiologic data of this rare neoplasm be assessed by a prospective, multi-center study in the future.

In conclusion, EWDAs were endoscopically ambiguous elevated tumors with an undermining growth pattern and few ulcers. Mucosal glandular cancerization and submucosal lobular endocervical glandular hyperplasia-like growth pattern were frequently observed histologic patterns. The microscopic features such as irregular glandular shapes, inharmonious glands, glands with ample mucin distension, and undulating apical border were frequently observed in EWDAs. For the timely diagnosis of these deeply seated tumors, generous forceps biopsies are recommended.

\section{Supplementary Information}

The Data Supplement is available with this article at https://doi.org/10.4132/ jptm.2021.10.12.

\section{Ethics Statement}

Our study was approved by the Institutional Review Board of the Asan Medical Center (\#2021-0485) with a waiver of the requirement for informed consent.

\section{Availability of Data and Material}

The datasets generated or analyzed during the study are available from the corresponding author on reasonable request.

\section{Code Availability}

Not applicable.

\section{ORCID}

Jongwon Lee https://orcid.org/0000-0003-3057-7874

In-Seob Lee https://orcid.org/0000-0003-3099-0140

Ji Yong Ahn https://orcid.org/0000-0002-0030-3744

Young Soo Park https://orcid.org/0000-0001-5389-4245

Jihun Kim https://orcid.org/0000-0002-8694-4365

\section{Author Contributions}

Conceptualization: JL, JK, YSP, ISL, JYA. Data curation: JK, JL. Methodology: JL, JK, YSP, ISL, JYA. Writing—original draft: JL, JK. Writing-review \& editing: JK, JL. Approval of final manuscript: all authors.

\section{Conflicts of Interest}

The authors declare that they have no potential conflicts of interest.

\section{Funding Statement}

No funding to declare.

\section{References}

1. Yao T, Utsunomiya T, Oya M, Nishiyama K, Tsuneyoshi M. Extremely well-differentiated adenocarcinoma of the stomach: clinicopathological and immunohistochemical features. World J Gastroenterol 2006; 12: 2510-6.

2. Joo M, Han SH. Gastric-type extremely well-differentiated adenocarcinoma of the stomach: a challenge for preoperative diagnosis. J Pathol Transl Med 2016; 50: 71-4.

3. Kim KM, Bilous M, Chu KM, et al. Human epidermal growth factor receptor 2 testing in gastric cancer: recommendations of an Asia-Pacific task force. Asia Pac J Clin Oncol 2014; 10: 297-307.

4. Jeong JH, Kim J, Hong YS, et al. HER2 amplification and cetuximab efficacy in patients with metastatic colorectal cancer harboring wildtype RAS and BRAF. Clin Colorectal Cancer 2017; 16: e147-52.

5. Hong YS, Kim J, Pectasides E, et al. Src mutation induces acquired lapatinib resistance in ERBB2-amplified human gastroesophageal adenocarcinoma models. PLoS One 2014; 9: e109440.

6. Waters KM, Salimian KJ, Voltaggio L, Montgomery EA. Refined criteria for separating low-grade dysplasia and nondysplastic Barrett esophagus reduce equivocal diagnoses and improve prediction 
of patient outcome: a 10-year review. Am J Surg Pathol 2018; 42: 1723-9.

7. WHO Classification of Tumours Editorial Board. WHO classification of tumours of the digestive system tumours. Lyon: IARC Press, 2019.

8. Humphris JL, Jones DB. Subepithelial mass lesions in the upper gastrointestinal tract. J Gastroenterol Hepatol 2008; 23: 556-66.
9. Niimi C, Goto H, Ohmiya N, et al. Usefulness of p53 and Ki-67 immunohistochemical analysis for preoperative diagnosis of extremely well-differentiated gastric adenocarcinoma. Am J Clin Pathol 2002; 118: 683-92.

10. Nakayama T, Ling ZQ, Mukaisho K, Hattori T, Sugihara H. Lineage analysis of early and advanced tubular adenocarcinomas of the stomach: continuous or discontinuous? BMC Cancer 2010; 10: 311. 